\title{
Analysis of Least-squares State Estimators for a Harmonic Oscillator
}

\author{
Michael Bask and Alexander Medvedev ${ }^{1}$
}

\begin{abstract}
The concept of least-squares observer is revisited. Robustness properties of this class of observers with respect to norm-bounded measurement noise are investigated and shown to be very much dependent on the operator chosen for the observer implementation. For the case of a harmonic oscillator, an explicit observer parameterization in terms of the implementation operator and the oscillator frequency is obtained, observer's existence conditions are proven and analyzed.
\end{abstract}

\section{Introduction}

A very common topic today is vibration and noise. Vibrations and noise arise in all types of constructions and therefore often worsen our working environment and/or reduce the standard of living.

A very common reason for vibration is a rotating unbalance. This kind of disturbances is special since it is often possible to measure or estimate the angular velocity with high accuracy. Then the measurement or estimate of the frequency can be used as a parameter in a controller to cancel or isolate the vibration.

The two disturbance problems, vibration and noise, are closely related to each other and can be termed as periodic disturbances. However, the period of a disturbance is very likely to change in time due to altering working conditions. In such a case, using active methods to control the vibration $[1,2]$ is a promising way to deal with the problem.

When not only the period but as well the signal form of the disturbance is known, a regular sinus function (or a number of those) can be utilized to model the disturbance. Thich leads to a harmonic oscillator.

Two control structures for disturbance rejection have been drawing most attention, the Internal Model Controller (IMC) and the External Model Controller (EMC). The difference between the control structures is that the IMC has the model of the disturbance included in the feedback loop and is therefore excited by the control error signal. The EMC treats the disturbance as an autonomous system which has no input. This naturally assumes that the disturbance can be described as an autonomous system. If the disturbance is observable from the output, all the states of the model can be reconstructed and, via a

${ }^{1}$ Control Engineering Group, Luleå University of Technology, S-971 87 Luleå, SWEDEN; fax: +46 920915 58; email: $\{$ Michael.Bask, Alexander.Medvedev\}@sm.luth.se state feedback, fed back to the plant. It was first introduced by Tomizuka, [3] and has been generalized to a MIMO-case in $[4,5]$.

Together with the EMC in $[4,5,6]$, a Least-Squares Deadbeat Observer is used. In its general form, this state estimator has been given in [7]. Compared to a usual FIR-filter, it contains a bank of pseudodifferential operators whose parameters can be chosen e. g. to enhance observer's robustness against plant parameters variation and/or measurement noise.

The purpose of this paper is to shed more light on the design aspects of least-squares state estimation, particularly for the practically important case of the harmonic oscillator, keeping in mind its perspective application in active vibration or noise control systems.

The article proceeds as follows. First, a brief introduction to least-squares state estimation is provided. Then, the issue of observer robustness against measurement disturbances is discussed. Next, new parameterizations of the least-squares observer for the harmonic oscillator are presented and analyzed. It follows by a line of action for determining the design parameters in the least-squares observer for the harmonic oscillator. A evaluation of Fréchet derivative and a simulation example illustrates the observer's robustness with respect to a structured parameter perturbation.

\section{Least-squares state estimator}

Consider the autonomous system

$$
\begin{aligned}
& \dot{x}(t)=F x(t) \\
& y(t)=C x(t)
\end{aligned}
$$

where $x(t) \in \mathrm{R}^{n}$ is the state vector with the initial condition $x(0)=x_{0}, y(t) \in \mathrm{R}^{\ell}$ is the output vector, the eigenvalues of $F$ are $\sigma(F)=\left\{\mu_{1}, \ldots, \mu_{n}\right\}$.

Let the operator $(P \cdot)(\lambda ; t)$, depending on parameter $\lambda \in \Lambda$ be defined via the Laplace inversion integral

$$
(P v)(\lambda ; t)=\frac{1}{2 \pi j} \int_{c-\infty}^{c+\infty} p(\lambda, s) V(s) e^{s t} d s
$$

where $V(s)=(\mathcal{L} v)(s)$ and $c$ is a suitable real constant and $\Lambda$ is a nonempty real set of cardinality $k$.

Suppose that $p(\lambda, s)$ is analytic everywhere inside any finite nonempty domain, except perhaps for the points of removable singularities. Moreover, let the following two conditions hold. 
Assumption 1 There exist real constants $c, \tau>0$ such that the inequality

$$
\left|p\left(\lambda, c+R e^{j \varphi}\right)\right| \leq e^{-(c+R \cos \varphi) \tau} ; \frac{\pi}{2} \leq \varphi \leq \frac{2 \pi}{3}
$$

holds for any large enough $R>0$.

Assumption 2 For any given $\lambda \in \Lambda$ and $\mu_{i} \in \sigma(F)$, it holds that

(i) $p\left(\mu_{i}\right) \neq p\left(\mu_{j}\right)$ if $\mu_{i} \neq \mu_{j}$

(ii) $\left.\frac{d p(s)}{d s}\right|_{s=\mu_{i}} \neq 0$ for every eigenvalue $\mu_{i}$ with height of the Jordan block greater than 1.

Consider now the following observer

$$
\hat{x}(t)=\mathcal{V}^{-1} \sum_{\lambda_{i} \in \Lambda} p\left(\lambda_{i}, F^{T}\right) C^{T} P(y)\left(\lambda_{i} ; t\right)
$$

where

$$
\mathcal{V}=\sum_{\lambda_{i} \in \Lambda} p\left(\lambda_{i}, F\right)^{T} C^{T} C p\left(\lambda_{i}, F\right)
$$

As demonstrated in [8], there is always a state-space realization of (1) such that the gramian matrix $\mathcal{V}$ is equal to a unit matrix. In the sequel, the system equations are assumed to be in a $\mathcal{V}$-balanced realization, if not stated otherwise.

Regarding observer (4), Assumption 1 specifies what kind of operators $P$ can be used for the least-squares state estimation while Assumption 2 relates the design parameters $\lambda_{i} \in \Lambda$ used in it to the spectral structure of the plant model $\sigma(F)$.

When assumptions 1,2 are fulfilled, observer (4) possesses a deadbeat performance, in the sense that $\hat{e}(t)=x(t)-\hat{x}(t) \equiv 0 ; t \geq \tau$ for any $x_{0}$. Furthermore, the minimal number of $\lambda_{i} \in \Lambda$ for which the observer always exists is equal to $n,[8]$.

Depending on the operator in question, $\lambda$ can either be integer and mean operator multiplicity or just a real parameter. Three examples of pseudodifferential operators used in the literature for designing state estimators are given in Table 1 . The use of the differential operator $P_{d}$ is classical (e.g. [9]), while the time delay operator $P_{\tau}$ is a more practical choice $[10,11]$. More recently, it has been shown how measurement disturbance attenuation in the least-squares state estimation can be improved by employing the slidingwindow convolution operator $P_{c},[8,12]$.

Table 1: Operators Used in Least-Squares State Estimation.

\begin{tabular}{|l|c|l|}
\hline \hline Operator & mapping & symbol \\
\hline$\left(P_{d} v\right)(\lambda ; t)$ & $v \stackrel{P_{d}}{\rightarrow} \frac{d^{\lambda} v}{d t^{\lambda}}$ & $s^{\lambda}$ \\
\hline$\left(P_{\tau} v\right)(\lambda ; t)$ & $v \stackrel{P_{\tau}}{\rightarrow} v(t-\lambda)$ & $e^{-\lambda s}$ \\
\hline$\left(P_{c} v\right)(\lambda, \tau ; t)$ & $v \stackrel{P_{c}}{\rightarrow} \int_{t-\tau}^{t} e^{\lambda(t-\theta)} v(\theta) d \theta$ & $\frac{1-e^{(\lambda-s) \tau}}{s-\lambda}$ \\
\hline \hline
\end{tabular}

\section{Robustness against measurement disturbance}

Assume now that the output signal in (1) is corrupted by a bounded disturbance $v \in \mathrm{L}_{2}^{\ell}, i$. $e$.

$$
y(t)=C x(t)+v(t)
$$

The state estimation error of observer (4) is

$$
\begin{aligned}
e(t) & =x(t)-\hat{x}(t) \\
& =x(t)-\sum_{\lambda_{i} \in \Lambda} p\left(\lambda_{i}, F^{T}\right) C^{T} P(y)\left(\lambda_{i} ; t\right) \\
& =x(t)-\sum_{\lambda_{i} \in \Lambda} p\left(\lambda_{i}, F^{T}\right) C^{T}\left(C P(x)\left(\lambda_{i} ; t\right)+P(v)\left(\lambda_{i} ; t\right)\right)
\end{aligned}
$$

From [7], it is known that

$$
P(x)\left(\lambda_{i} ; t\right)=p\left(\lambda_{i}, F\right) x(t)
$$

Thus, the observer estimation error can be written as

$$
e(t)=-\sum_{\lambda_{i} \in \Lambda} p\left(\lambda_{i}, F^{T}\right) C^{T} P(v)\left(\lambda_{i} ; t\right)
$$

or, in a more concise form

$$
e(t)=-W_{k}^{T} E_{k}(t)
$$

where

$$
W_{k}=\left[\begin{array}{c}
C p\left(\lambda_{1}, F\right) \\
\vdots \\
C p\left(\lambda_{k}, F\right)
\end{array}\right] ; E_{k}(t)=\left[\begin{array}{c}
P(v)\left(\lambda_{1} ; t\right) \\
\vdots \\
P(v)\left(\lambda_{k} ; t\right)
\end{array}\right]
$$

The form of (5) implies two possibilities of minimizing the observer estimation error, either by exploiting geometric properties of $W_{k}$ or by changing the parameter set $\Lambda$.

The vector $E_{k}$ can be completely defined by its orthogonal projections onto Ker $W_{k}^{T}$ and onto $\operatorname{Im} W_{k}$ which are respectively given by

$$
\begin{aligned}
\Pi_{\mathrm{Ker}} E_{k} & =\left(I-\left(W_{k}^{T}\right)^{+} W_{k}^{T}\right) E_{k} \\
\Pi_{\mathrm{Im}} E_{k} & =\left(W_{k}^{T}\right)^{+} W_{k}^{T} E_{k}
\end{aligned}
$$

where $(\cdot)^{+}$denotes pseudoinverse.

Clearly, $\Pi_{\text {Ker }} E_{k}$ has no effect on the estimation error and, therefore, $W_{k}$ should be constructed so that $\left|\Pi_{\mathrm{Ker}} E_{k}\right|$ is maximized. Unfortunately, for an unstructured disturbance $v$, there is no other way to pursue this approach but increasing the cardinality of $\Lambda$. Whenever observer (4) exists, $\operatorname{dim} \operatorname{Ker} W_{k}^{T}=$ $k \ell-n$ and, then, using more operators $P(\cdot)\left(\lambda_{i} ; t\right), i=$ $1, \ldots, k$ enlarges the subspace Ker $W_{k}^{T}$ and improves the chances for better accommodation of the disturbance signal in it.

The second disturbance component, $\Pi_{I m} E_{k}$, has to be dealt with via filtering properties of the operators $P(\cdot)\left(\lambda_{i} ; t\right)$. 
Let $x_{i} \in \mathrm{L}_{2}, i=1, \cdots, n$ form a vector $x=$ $\left[x_{1}, \cdots, x_{n}\right]^{T}$. Using the conventional notation for the signal norms

$$
\begin{aligned}
& \left\|x_{i}\right\|_{\infty}=\operatorname{ess} \sup _{t}\left|x_{i}\right| ;\|x\|_{\infty}=\max _{i}\left\|x_{i}\right\|_{\infty} \\
& \|x\|_{2}=\left(\int_{-\infty}^{\infty} x^{T}(t) x(t) d t\right)^{\frac{1}{2}}
\end{aligned}
$$

and $|\cdot|_{2},|\cdot|_{\infty},|\cdot|_{1}$ for the usual matrix norms, the following upper bounds on the estimation error $e$ can be derived.

Theorem 1 Suppose Assumption 1 and Assumption 2 are satisfied. Then, with respect to (5), the following inequalities hold

$$
\begin{aligned}
\|e(t)\|_{2} & \leq\left(\sum_{i=1}^{r}\left\|p\left(\lambda_{i}, s\right)\right\|_{\infty}^{2}\right)^{\frac{1}{2}}\|v(t)\|_{2} \\
\|e(t)\|_{\infty} & \leq\left|W_{k}\right|_{1} \max _{i=1, \ldots, k}\left\|p\left(\lambda_{i}, s\right)\right\|_{2}\|v(t)\|_{2}
\end{aligned}
$$

Proof: Partition $E_{k}(t)$ in the following way

$$
E_{k}(t)=\left[\begin{array}{c}
E_{1, k} \\
\vdots \\
E_{k, k}
\end{array}\right] ; E_{i, k}=(P v)\left(\lambda_{i} ; t\right)
$$

Direct evaluation of 2 -norm of the state estimation error gives

$$
\begin{aligned}
\|e(t)\|_{2}^{2} & =\int_{-\infty}^{\infty} E_{k}^{T}(t) W_{k} W_{k}^{T} E_{k}(t) d t \\
& \leq\left|W_{k} W_{k}^{T}\right|_{2} \int_{-\infty}^{\infty} E_{k}^{T}(t) E_{k}(t) d t
\end{aligned}
$$

Since the system realization is $\mathcal{V}$-balanced, the columns of $W_{k}$ are orthogonal and normalized vectors in $R^{k \ell}$ and then

$$
\begin{aligned}
\left|W_{k} W_{k}^{T}\right|_{2} & =\max _{i=1, \ldots, k \ell} \mu_{i}^{\frac{1}{2}}\left(W_{k} W_{k}^{T}\right) \\
& =\max _{i=1, \ldots, n} \mu_{i}^{\frac{1}{2}}\left(W_{k}^{T} W_{k}\right)=1
\end{aligned}
$$

where $\mu_{i}(\cdot)$ denotes an eigenvalue of the matrix.

Proceeding with the inequality above

$$
\begin{aligned}
\|e(t)\|_{2} \leq\left\|E_{k}(t)\right\|_{2} & =\left(\sum_{i=1}^{k}\left\|E_{i, k}\right\|_{2}^{2}\right)^{\frac{1}{2}} \\
& \leq\left(\sum_{i=1}^{r}\left\|p\left(\lambda_{i}, s\right)\right\|_{\infty}^{2}\right)^{\frac{1}{2}}\|v\|_{2}
\end{aligned}
$$

In the last step, a well known system gain [13] is used

$$
\left\|E_{i, k}\right\|_{2}^{2} \leq\left\|p\left(\lambda_{i}, s\right)\right\|_{\infty}^{2}\|v\|_{2}
$$

along with the fact that $\left\|P(\cdot)\left(\lambda_{i} ; t\right)\right\|_{\infty}=\left\|p\left(\lambda_{i}, s\right)\right\|_{\infty}$ This completes the proof for $(6)$.

In order to prove (7), consider $\infty$-norm of (5)

$$
\|e(t)\|_{\infty} \leq\left|W_{k}^{T}\right|_{\infty}\left\|E_{k}(t)\right\|_{\infty}=\left|W_{k}\right|_{1}\left\|E_{k}(t)\right\|_{\infty}
$$

Utilizing another $\left(\|\cdot\|_{2}\right.$ to $\left.\|\cdot\|_{\infty}\right)$ system gain [13] gives

$\left\|E_{i, k}\right\|_{\infty} \leq\left\|(P \cdot)\left(\lambda_{i} ; t\right)\right\|_{2}\|v(t)\|_{2}=\left\|p\left(\lambda_{i}, s\right)\right\|_{2}\|v(t)\|_{2}$

The equality is due to Parseval's theorem. Combining two inequalities above yields (7).

Taking advantage of the results obtained in Theorem 1 , one can carry out a comparison of disturbance rejection properties among the operators in Table 1. All necessary operator norms are evaluated in [14] and shown in Table 2.

Noteworthy, the differential operator $P_{d}$ has infinite 2 - and $\infty$-norms which makes it possible for $e$ to rise indefinitely. Therefore, pure differentiation should be avoided in state estimation.

Turning to the delay operator $P_{\tau}$, one notices that its $\infty$-norm is finite (actually, $\left\|P_{\tau}\right\|_{\infty}=1$ ), whilst 2 -norm is yet infinite. Logically, in this case, a 2norm bounded disturbance must produce a 2-norm bounded response in the estimation error. Unfortunately, this does not always guarantee a good design. For example, in such a design, a Dirac delta like disturbance would cause a Dirac delta like response in the state estimates, which is an undesirable feature for an observer to have.

From Theorem 1, it becomes evident that an appropriate, for observer implementation, operator has to possess both finite $\infty$ - and 2-norms. All these properties have been proven for the operator $P_{c}$.

Table 2: Operator norms.

\begin{tabular}{|l|c|l|}
\hline \hline Operator & $\|\cdot\|_{2}$ & $\|\cdot\|_{\infty}$ \\
\hline$\left(P_{d} v\right)(\lambda ; t)$ & $\infty$ & $\infty$ \\
\hline$\left(P_{\tau} v\right)(\lambda ; t)$ & $\infty$ & 1 \\
\hline$\left(P_{c} v\right)(\lambda, \tau ; t)$ & $\sqrt{\frac{e^{2 \lambda \tau}-1}{2 \lambda}}$ & $\frac{e^{\lambda \tau}-1}{\lambda}$ \\
\hline \hline
\end{tabular}

Regarding the operator $P_{c}$, it is worth to notice that $\lambda$ and $\tau$ in it can be evaluated in terms of $\left\|p_{c}\right\|_{2}$ and $\left\|p_{c}\right\|_{\infty}$

$$
\begin{aligned}
\tau & =\frac{2\|p\|_{2}^{2}-\mathrm{e}^{2}\|p\|_{\infty}}{\mathrm{e}^{2}-1} \ln \left(\frac{2\|p\|_{2}^{2}-\|p\|_{\infty}}{2\|p\|_{2}^{2}-\mathrm{e}^{2}\|p\|_{\infty}}\right) \\
\lambda & =\frac{\mathrm{e}^{2}-1}{2\|p\|_{2}^{2}-\mathrm{e}^{2}\|p\|_{\infty}}
\end{aligned}
$$

Since $\tau$ has to be positive, one of the two following inequalities should hold 


$$
\begin{array}{r}
0<\|p\|_{2}^{2}<\frac{\|p\|_{\infty}}{2} \\
\|p\|_{2}^{2}>\frac{\mathrm{e}^{2}}{2}\|p\|_{\infty}
\end{array}
$$

\section{Least-squares state estimator for a harmonic oscillator}

Consider now a harmonic oscillator, i. e. autonomous dynamic system (1) whose matrices are the following ones

$$
\begin{aligned}
& F=\left[\begin{array}{cc}
0 & \omega^{2} \\
-1 & 0
\end{array}\right] \\
& C=\left[\begin{array}{ll}
1 & 1
\end{array}\right]
\end{aligned}
$$

Theorem 2 Assume that for any $\lambda \in \Lambda$, the operator $P(\cdot)(\lambda ; t)$ satisfies Assumption 1. Then, the observer

$$
\hat{x}(t)=U \mathcal{V}_{F}^{-1} \sum_{\lambda \in \Lambda}\left[\begin{array}{c}
p(j \omega, \lambda) P(y)(\lambda ; t) \\
p(-j \omega, \lambda) P(y)(\lambda ; t)
\end{array}\right]
$$

where

$$
\begin{aligned}
U & =\left[\begin{array}{cc}
\frac{-j \omega}{1-j \omega} & \frac{j \omega}{1+j \omega} \\
\frac{1}{1-j \omega} & \frac{1}{1+j \omega}
\end{array}\right] ; \\
\mathcal{V}_{F} & =\sum_{\lambda \in \Lambda}\left[\begin{array}{cc}
p^{2}(j \omega, \lambda) & p(j \omega, \lambda) p(-j \omega, \lambda) \\
p(j \omega, \lambda) p(-j \omega, \lambda) & p^{2}(-j \omega, \lambda)
\end{array}\right]
\end{aligned}
$$

yields the estimate $\hat{x}(t)=x(t), t>\tau$ if and only if there is no $c \in \mathrm{C}$ such that

$$
p(j \omega, \lambda)=c p(-j \omega, \lambda), \forall \lambda \in \Lambda
$$

Proof: For the values of the system parameters given in $(8), p(F, \lambda)$ can be evaluated as the following integral over a contour enclosing $\pm j \omega$

$$
p(F, \lambda)=\frac{1}{2 j \pi} \oint p(s, \lambda)(s I-F)^{-1} d s
$$

A direct application of the Residue Theorem results in

$$
p(F, \lambda)=\frac{1}{2}\left[\begin{array}{ll}
p_{11} & p_{12} \\
p_{21} & p_{11}
\end{array}\right]
$$

where

$$
\begin{aligned}
& p_{11}=p(j \omega, \lambda)+p(-j \omega, \lambda) \\
& p_{12}=-j \omega(p(j \omega, \lambda)-p(-j \omega, \lambda)) \\
& p_{21}=\frac{1}{j \omega}(p(-j \omega, \lambda)-p(j \omega, \lambda))
\end{aligned}
$$

Further

$$
C p(F, \lambda)=[p(j \omega, \lambda) \quad p(-j \omega, \lambda)] U^{-1}
$$

were

$$
U^{-1}=\frac{1}{2}\left[\begin{array}{ll}
1-\frac{1}{j \omega} & 1-j \omega \\
1+\frac{1}{j \omega} & 1+j \omega
\end{array}\right]
$$

Substituting the expression for $C p(F, \lambda)$ into (4) renders (9).

It is easy to see that for the case in hand

$$
\mathcal{V}=U^{-T} \mathcal{V}_{F} U^{-1}
$$

The matrix $U$ is always nonsingular since

$$
\operatorname{det}\left(U^{-1}\right)=2 j\left(\omega+\frac{1}{\omega}\right)
$$

Cauchy's inequality for $p(j \omega, \lambda)$ and $p(-j \omega, \lambda)$ reads

$$
\left(\sum_{\lambda \in \Lambda} p(j \omega, \lambda) p(-j \omega, \lambda)\right)^{2} \leq \sum_{\lambda \in \Lambda} p^{2}(j \omega, \lambda) \sum_{\lambda \in \Lambda} p^{2}(-j \omega, \lambda)
$$

where equality occurs iff (10). Noticing that

$$
\begin{aligned}
\operatorname{det} \mathcal{V}_{F} & =\sum_{\lambda \in \Lambda} p^{2}(j \omega, \lambda) \sum_{\lambda \in \Lambda} p^{2}(-j \omega, \lambda) \\
& -\left(\sum_{\lambda \in \Lambda} p(j \omega, \lambda) p(-j \omega, \lambda)\right)^{2}
\end{aligned}
$$

completes the proof.

In practice, mostly the time delay operator is used for the least-squares observer implementation. Thus, this simple case deserves special consideration.

Corollary 1 For $p(s, \lambda)=\mathrm{e}^{-s \lambda}, \Lambda=\left\{\lambda_{1}, \ldots, \lambda_{k}\right\}$, observer (9) takes the form

$$
\hat{x}(t)=\sqrt{1+\omega^{2}} \mathcal{V}^{-1} \sum_{\lambda \in \Lambda} V(\lambda) y(t-\lambda)
$$

where $\alpha=\arctan \omega$ and

$$
\begin{aligned}
& V(\lambda)=\left[\begin{array}{c}
\omega \sin (\omega \lambda+\alpha) \\
\cos (\omega \lambda+\alpha)
\end{array}\right] \\
& \mathcal{V}=\frac{1+\omega^{2}}{2}\left[\begin{array}{ll}
v_{11} & v_{12} \\
v_{12} & v_{22}
\end{array}\right]
\end{aligned}
$$

$$
\begin{aligned}
& v_{11}=\frac{1}{\omega^{2}}\left(k-\sum_{\lambda \in \Lambda} \cos (2 \omega \lambda+2 \alpha)\right) \\
& v_{12}=\frac{1}{\omega} \sum_{\lambda \in \Lambda} \sin (2 \omega \lambda+2 \alpha) \\
& v_{22}=k+\sum_{\lambda \in \Lambda} \cos (2 \omega \lambda+2 \alpha)
\end{aligned}
$$


Proof: The proof is straightforward by evaluation of the matrix exponential of $F$

$$
\exp (-F \lambda)=\left[\begin{array}{cc}
\cos (\omega \lambda) & -\omega \sin (\omega \lambda) \\
\frac{1}{\omega} \sin (\omega \lambda) & \cos (\omega \lambda)
\end{array}\right]
$$

and the corresponding matrices $\exp \left(-F^{T}\right) C^{T}, \mathcal{V}$.

When the time delays in $\Lambda$ are taken to be commensurate $i . e . \lambda_{p}=p T, p=1, \ldots, k$ for some $T>0$, the elements of matrix $\mathcal{V}$ in (9) have simpler form

$$
\begin{aligned}
& v_{11}=\frac{1}{\omega^{2}}\left(k+\frac{\sin \omega k T}{\sin \omega T} \sin (\omega T(k+1)-\beta)\right) \\
& v_{12}=\frac{\sin \omega k T}{\omega \sin \omega T} \cos (\omega T(k+1)-\beta) \\
& v_{22}=k-\frac{\sin \omega k T}{\sin \omega T} \sin (\omega T(k+1)-\beta)
\end{aligned}
$$

where $\beta=\arctan \frac{1-\omega^{2}}{2 \omega}$. The determinant of $\mathcal{V}$ is also easy to derive

$$
\operatorname{det} \mathcal{V}=\frac{\left(1+\omega^{2}\right)^{2}}{4 \omega^{2}}\left(k^{2}-\frac{\sin ^{2} \omega k T}{\sin ^{2} \omega T}\right)
$$

For each value of $k$, there is a condition on $\omega T$ that guarantees det $\mathcal{V} \neq 0$. For instance, choosing $T$ so that $\sin \omega T \neq 0$ does the trick for the case of two $\lambda$. It is easy to see that the following inequality holds for all $k$

$$
k^{2} \geq \frac{\sin ^{2} \omega k T}{\sin ^{2} \omega T}
$$

Indeed, denote

$$
w_{k}=\frac{\sin \omega k T}{\sin \omega T}
$$

Apparently, for $k=1$ the inequality above holds and $w_{1}=1$. Further, $w_{k}$ obeys a simple recursion

$$
w_{k+1}=\cos \omega T w_{k}+\cos \omega k T
$$

Then

$$
w_{k+1}^{2} \leq(k+1)^{2}
$$

\section{Observer sensitivity to frequency variation}

Most of the properties of observer (4) are defined by the symbol of the pseudodifferential operator used for implementation. To investigate observer sensitivity to changes in the model parameters, it is reasonable to take advantage of so-called Fréchet derivative. For $p(F, \lambda)=\exp (-F \lambda)$, it leads to the following expression

$$
S(Z)=\lim _{\delta \rightarrow 0} \frac{\exp (-(F+\delta Z) \lambda)-\exp (-F \lambda)}{\delta}
$$

where the derivative is taken at $Z$

$$
Z=\left[\begin{array}{ll}
0 & 1 \\
0 & 0
\end{array}\right]
$$

The special structure of $Z$ is due to the fact that only $\omega$ can change in (8).

A straightforward calculation shows that

$$
\begin{array}{r}
\|S(Z)\|_{\infty}=\max \left(\left|\frac{(1-\omega \lambda) \cos (\omega \lambda)}{w^{2}}\right|+|\lambda \sin (\omega \lambda)|,\right. \\
|\lambda \sin (\omega \lambda)|+|\omega \lambda \cos (\omega \lambda)+\sin (\omega \lambda)|)
\end{array}
$$

By choosing $\lambda$ minimizing the value of $\|S(Z)\|_{\infty}$, minimum sensitivity of the matrix exponential, and therefore for the whole observer, with respect to oscillator frequency variations is achieved.

Two observers with different choices of $\lambda$ are designed, one with good (w. r. t. the value of $\left.\|S(Z)\|_{\infty}\right)$ choices of $\lambda, \lambda=\{0.001,0.013\}$ and one with bad choices, $\lambda=\{0.043,0.044\}$. The estimation error for each of the observers is plotted in Fig. (1). There, it can be seen that an appropriate choice of the design parameters decreases the estimation error due to frequency mismatch.

\section{Simulation example}

The process is oscillator (1) with $\omega=100$. The purpose of simulation is to investigate the observer robustness against variation of $\omega$ when utilizing $P_{\tau}$ and $P_{c}$. Both observers use only two operators $P(\cdot)(\lambda ; t)$. For $P_{d}$, the design parameters are chosen as $\Lambda=\{0.001,0.013\}, \Lambda=\{0.043,0.044\}$, while for $P_{c}$, the time constants are $\Lambda=\{-30,-35\}$ and $\tau=0.01$.

In Fig. 1, a comparison of the estimation error norm $|e|_{2}$ for observer (11), and a one of the form (9) is presented. It can be clearly seen that the observer implemented by means of the finite-memory convolution operator performs better both in the transient response and in the steady-state.
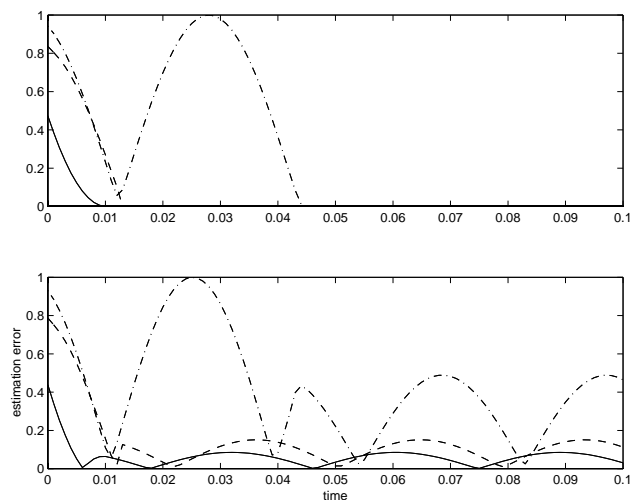

Figure 1: $|e|_{2}$ for the observer with $P_{d}$ and good $\lambda$ (dashed line), bad $\lambda$ (dashdotted line) and for the observer with $P_{c}$ (solid line). Upper figure - no perturbation and lower figure perturbation in $\omega 10 \%$.

Since the estimation error is periodic, after the transient time is expired, the following quadratic perfor- 
mance criterion is introduced

$$
\|e\|_{T}=\frac{1}{T} \int_{0}^{T} e^{T}(t) e(t) d t
$$

where the integral is taken over an estimation error signal period $T$. Fig. 2 gives an idea why very large values of $k$ are needed in applications in order to achieve reasonably low observer sensitivity when the time delay operator is used (the usual FIR-filter). Notice that a logarithmic scale is used to make the figure informative.

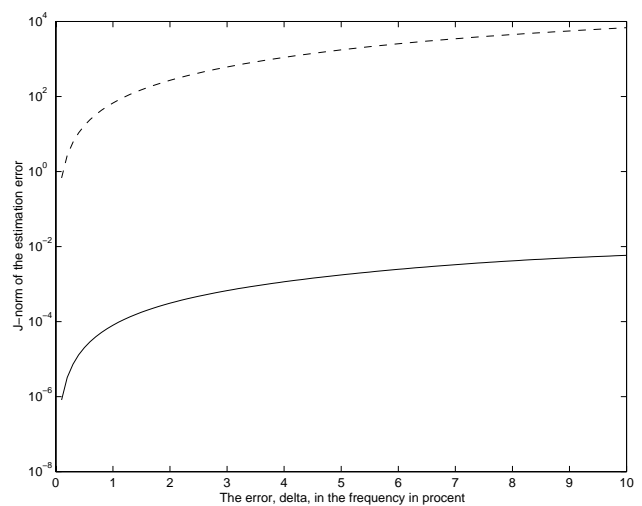

Figure 2: $\|e\|_{T}$ for the observer with $P_{d}$ (dotted line) and for the observer with $P_{c}$ (solid line) w. $r$. $t$. the frequency perturbation in percent.

\section{Conclusion}

For a broad class of least-squares observers, upper bounds on the estimation error for measurements subject to $\mathrm{L}_{2}$-disturbance are derived. It is demonstrated that the choice of the implementation operator plays a crucial role in the observer design. A practically important case of state estimation of a harmonic oscillator is investigated, new parameterizations of the least-squares observer in terms of the oscillator's frequency together with observer existence conditions are presented and proven. A simulation example illustrates that observer robustness properties against a structured uncertainty are closely connected with the choice of the parameterization operator.

\section{Acknowledgement}

Financial support by Volvo Research Foundation and Swedish Research Council for Engineering Sciences is gratefully acknowledged.

\section{References}

[1] C.R. Fuller, S.J. Elliott, and P.A. Nelson, $A c-$ tive Control of Vibration, Academic Press Inc., 1996. [2] S.M. Kuo and D.R. Morgan, Active Noise Control Systems, John Wiley and Sons, Inc., 1996.
[3] M. Tomizuka, K.-K. Chew, and W.-C. Yang, "Distrubance rejection through an external model", ASME J. Dynamic Systems, Measurement, and Control, vol. 6, pp. 559-564, 1990.

[4] M. P. Bask and A. Medvedev, "External model controller for active vibration isolation", in Proceedings of MOVIC-98, The Fourth International Conference on Motion and Vibration Control, 1998.

[5] A. Medvedev and G. Hillerström, "An external model control system", Control-Theory and Advanced Technology, vol. 10, pp. 1643-1665, 1995.

[6] M.P. Bask and A. Medvedev, "External model reptitive controller for active vibration isolation", in In Proc. The 1999 Iinternational Symposium on Active Control of Sound and Vibration, 1999, pp. 177189 .

[7] A. Medvedev, "Continuous least-squares observers with applications", IEEE Transactions on automatic control, vol. 41, no. 10, pp. 1530-1537, October 1996.

[8] A. Medvedev, "State estimation and fault detection by a bank of continuous finite-memory filters", International Journal of Control, pp. 499-517, 1998

[9] Thomas Kailath, Linear Systems, PrenticeHall, Inc, 1980.

[10] A. Medvedev and H. Toivonen, "Feedforward time-delay structures in state estimation. Finite memory smoothing and continuous deadbeat observers", IEED, vol. 141, no. 2, pp. 121-129, 1994.

[11] D. H. Chyung, "State variable reconstruction", International Journal of Control, vol. 40, pp. 955963,1984 .

[12] A. Medvedev, "Disturbance attenuation in finite spectrum assignment controllers", Automatica, vol. 33, no. 6, pp. 1163-1168, June 1997.

[13] J. C. Doyle, B. A. Francis, and A. R. Tannenbaum, Feedback Control Theory, Macmillan Publishing Company, New York, 1992.

[14] A. Medvedev, "Disturbance attenuation enhancement in continuous parity space methods", in European Control Conference, Brussels, Belgium, July 1997, p. (available on CD). 